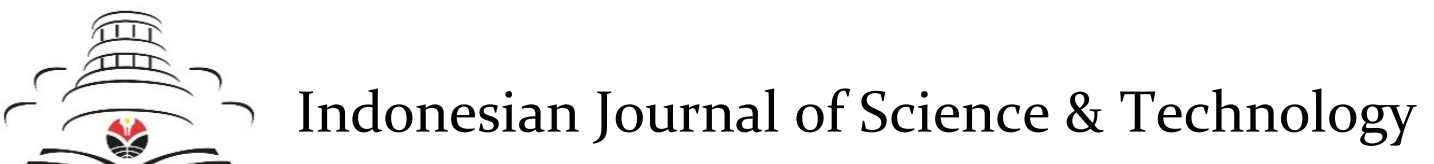

Journal homepage: http://ejournal.upi.edu/index.php/ijost/

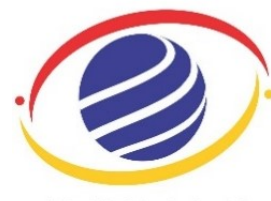

IJoST $T_{\text {Sciencese \& I Tournal of }}^{\text {Inology }}$

\title{
The Effect of Spoiler Shape and Setting Angle on Racing Cars Aerodynamic Performance
}

\author{
Hesam Eftekhari, Abdulkareem Sh. Mahdi Al-Obaidi", Shahrooz Eftekhari \\ School of Engineering, Taylor's University College, No. 1 Jalan SS 15/8 47500 Subang Jaya, Selangor DE, \\ Malaysia \\ *Correspondence: E-mail: Abdulkareem.mahdi@taylors.edu.my
}

\section{A B S TRACT}

Automotive racing is one of the favorite sports of human being. There have been many developments in past decades by car engineers to improve the performance of the engine and increase the aerodynamic efficiency of the race cars to achieve a better lap time and get a better placement safely. One of the ways to improve the aerodynamic performance of a race car is to use rear spoilers. This study by using ANSYS FLUENT numerically investigated the effect of the spoiler shape and setting angle on the aerodynamic characteristics of a race car and then it was validated by conducting wind tunnel experiment. Lift and drag coefficient of NACA0012, NACA4412, and S1223 are determined in Reynold's number of $2 \times 10^{5}$ as an airfoil and as spoiler on ERC model which is a conceptual car model inspired by Porsche 911. It was found that ERC model with spoiler would have better aerodynamic efficiency compared to ERC model without spoiler. Also, S1223 at -6 degrees was identified as the optimized configuration as it generates the highest downforce. Even though the drag coefficient at this setting angle is slightly higher, but in terms of stability and handling IT is at its best. Overall, this study would help car manufacturers, for racing and commercial purposes, to have a better insight into the effect of spoiler configuration on the aerodynamic performance of cars. Hence, the stability, handling, and efficiency of the cars can be further improved by selecting the suitable spoiler configuration.

\begin{tabular}{l}
\multicolumn{1}{c}{ A R T I C L E I N F O } \\
\hline Article History: \\
Submitted/Revised 05 Jul 2019 \\
First revised 29 Oct 2019 \\
Accepted 27 Jan 2020 \\
First available online 28 Jan 2020 \\
Publication date 01 Mar 2020 \\
\\
Keywords: \\
Aerodynamic Performance, \\
Spoiler, \\
Race Car, \\
CFD.
\end{tabular}




\section{INTRODUCTION}

Aerodynamic forces acting on a moving vehicle have a significant contribution to performance of the car in terms of fuel efficiency and stability (Walter, 2006). Drag force is one of the aerodynamic forces, which acts horizontally and opposes the forward motion of the vehicle when the vehicle is traveling which acts on the outside surface body of the vehicle that affect the fuel consumption and vehicle speed. Another important aerodynamic force is the lift force, which acts vertically and increases the grip of the car. In other words, it would increase the vehicle stability and handling.

Achieving a stable position and lap time during a race is one of the main challenges that car designers face. This requires them to achieve an optimum design for the cars to reduce the lap time by reducing the drag as well as maintaining the stability and maneuverability of the car by designing an optimum design for the car (Heisler, 2007). One method to improve the vehicle efficiency and stability is to add aerodynamic devices such as spoiler. Spoiler creates down force by creating a "dam" at rear lip of the trunk, which cause an improvement on the stability of a vehicle by decreasing lift and drag at high speed. Spoilers have different shapes and they can be set at different angles depending on the shape and design of the car. The spoilers help in enhancing the aerodynamic performance of cars by pressure reduction (Figure 1) and increase the stability of the race car during the race.

Buljac et al. (2016) focused on the effect of rear wing height which they investigated an airfoil-shape on 4 positions. Reynoldsaverage Navier-Stroke equations was used using k-epsilon turbulence model and standard wall function, with the assumption of steady viscous fluid flow.

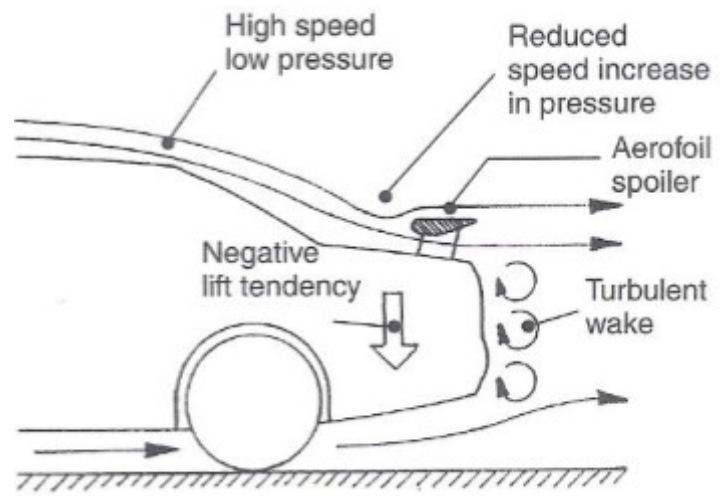

Figure 1. Negative lift generation using spoiler

Geometrical model of BMW E38 was used for the base body. Overall 5 different cases were studied, which were the vehicle without spoiler and the vehicle with spoiler at different heights. The spoiler was set on the rear boot of the vehicle and the spoiler itself had an airfoiled shape. Five different meshes were generated, and the front and rear wheel of the vehicle were simulated similar to Fackrell \& Harvey (1972). The road was defined as a moving wall with no slip and the only boundary layers were on the vehicle body. As the result, the rear spoiler increased the automobile drag and also increased the traction and stability. The best position for the rear spoiler was determined to be $39 \%$ of the highest between roof and rear boot, while the downforce to drag ratio was 0.57. Das \& Riyad (2017) studied on aerodynamic characteristics of racing cars to reduce the accidents because of wind loadings. From other side, it studied to reduce the fuel consumption. In this paper, a car body model and 2 different spoilers, one on rear boot, one which was fixed on roof with fixed angle, were generated using CAD software. Other than that, a wind tunnel was generated in CAD as well. Overall three different cases were studied, which were the car without spoiler, with first spoiler and with second spoiler. Based on the numerical analysis, which were conducted, it was concluded that the rear 
end spoiler provides more negative lift force than roof spoiler, while the drag reduction was less than roof spoiler. Yew et al. (2017) studied the initial prediction of the effects of aerodynamics of the performance of Taylor's formula SAE car. TR16 was studied, which was Taylor's Formula SAE car. The design of the car was generated in SOLIDWORKS and was simplified more using surface modelling to be able to generate a good mesh. The study findings showed that the front wing and diffuser produced higher lift to drag ratio compared to the original and base model with the value of 5.108 and 3.497 , respectively. It was concluded that the diffuser's capacity to produce downforce is mostly based on the inlet and outlet angle of it. Smaller inlet angle and larger outlet angle would give higher downforce and induced drag. As for the multi element, front wing reduced the drag induced by the tires of the car.

This paper mainly focused on the effect of different shapes of spoiler at different angles of attack ( $A O A)$ on the aerodynamic performance of a conceptual race car design named as ERC model. The model was inspired by Porsche 911 and it is selected based on collecting data and gathering information about Le Mans 24 hours race which is one of the most famous races. Based on the gathered data, the highest win belongs to the wellestablished car manufacturer, Porsche. The aerodynamic performance of the vehicle design in this study was numerically investigated using ANSYS FLUENT predicting the drag coefficient (CD) and lift coefficient (CL) on different spoiler shapes and setting angles. The numerical results were validated through experimental investigation using Taylor's University Wind Tunnel (TUWT).

\section{MATERIALS AND METHODS}

\subsection{Geometry Modelling}

The airfoils sections used for spoilers in the current study are NACA0012, NACA4412, and S1223. Literature shows that NACA 4412 and S1223 are one of the most commonly used airfoils for spoilers to improve the aerodynamic efficiency of race cars (Su \& Basavaraj, 2017; Vadgama, 2015). Comparison of the aerodynamic performance of these three helps to have a better understanding of the effect of camber on the aerodynamic characteristics of the spoiler since NACA 0012 is the symmetric airfoil, while NACA 4412 and S1223 are the positive and negative camber airfoils, respectively. The airfoil chord was calculated based on the TUWT test section size constraint to achieve the required Reynolds number. The chord length of all the test models is $0.015 \mathrm{~m}$ with span of $0.121 \mathrm{~m}$ and the height of the spoiler was set to have $39 \%$ of the distance between the boot to roof of the car model (Reddy \& Lokanadham, 2017).

As stated earlier, the design, and shape of this car model was inspired by Porsche 911. However, since the full model of 911 is too complex and meshing the car model is time consuming, a concept car model that is called ERC model was created in SOLIDWORKS, which is a simpler and modified version of Porsche 911 Carrera GTS. Table 1 presents the length, width, and height of ERC model (Figure 2).

Table 1. ERC Model Dimensions

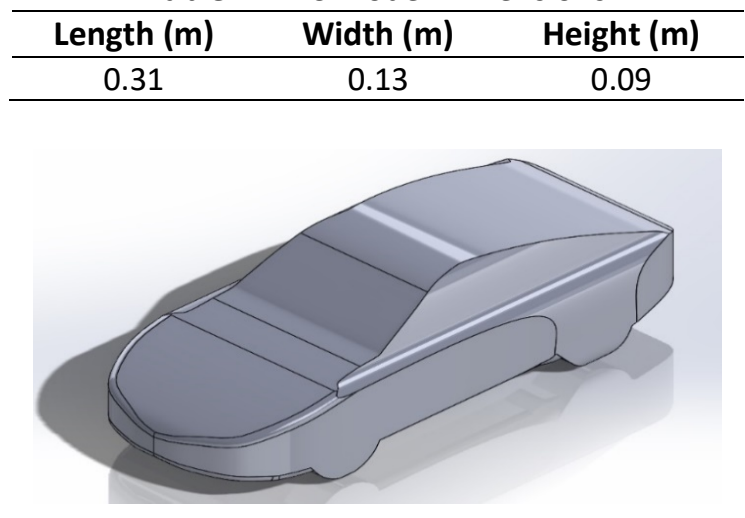

Figure 2. ERC CAD model 


\subsection{Numerical Method}

\subsubsection{Numerical Method}

One of the most applicable numerical methods is the computational fluid dynamics (CFD). Eftekhari \& Al-Obaidi (2019) boundary domain model and mesh generation method were used as a reference in this paper due to its efficiency and accuracy in prediction of aerodynamic forces. Yet there is a slight modification to the boundary domain, which is used for the ERC model with and without spoiler (Figure 3). Since the boundary domain need to be as close as the real conditions, the car model needs to be set on a straight surface (to replicate its condition on the road). The boundary domains are named as shown in Figure 4.

(a)

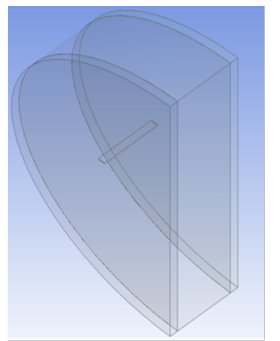

Figure 3. Boundary domain (a) and (b)

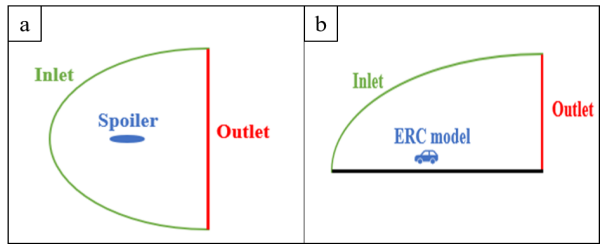

Figure 4. Boundary domain named selection: (a) spoiler and (b) ERC model

A fine and efficient mesh is required to obtain accurate simulation result. A C-type grid topology was used and applied in this research as it is identified to be an efficient mesh generation method for airfoil related geometries (Eftekhari \& Al-Obaidi, 2019). Sphere of influence was used for both Boundary A and Boundary B to concentrate the mesh refinement in areas nearby the

test model, which is the area around spoilers for Boundary A and ERC model for Boundary B. The mesh refinement was achieved by adjusting the edge sizing of the investigated geometries and inflation was applied to achieve better boundary layer capture during the simulation. The refined mesh obtained around NACA0012, NACA4412, and S1223 are shown in Figure 5.

(a)

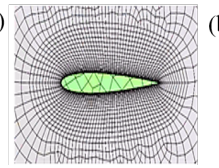

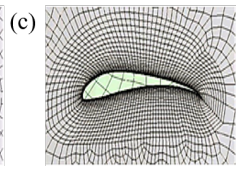

Figure 5. Spoilers mesh for NACA0012, NACA4412, and S1223

As for the mesh for ERC model without spoiler other than sphere of influence, face sizing and inflation were used. But, for ERC model with spoilers, because of the existence of spoiler, it is not possible to utilize the inflation. So, face sizing, edge sizing, and refinement were utilized to refine the mesh around the ERC model with spoiler. The generated mesh for ERC model without and with spoiler is shown in Figure 6. A summary of mesh characteristics is shown in Table 2.

(a)
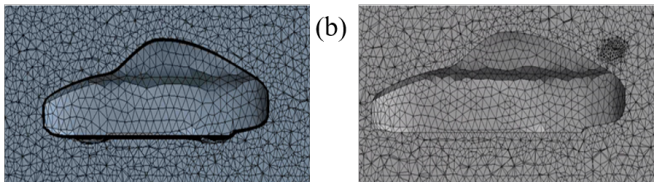

Figure 1. Mesh for ERC model: (a) without spoiler and (b) with NACA0012 spoiler

Table 2. Mesh Configuration

\begin{tabular}{llll}
\hline Model & Spoilers & $\begin{array}{l}\text { ERC with- } \\
\text { out spoiler }\end{array}$ & $\begin{array}{l}\text { ERC with } \\
\text { spoiler }\end{array}$ \\
\hline $\begin{array}{l}\text { Sphere of Influence } \\
\text { radius }(\mathrm{m})\end{array}$ & 0.25 & 0.6 & 0.6 \\
$\begin{array}{l}\text { Sphere element Size } \\
\text { Face sizing }\end{array}$ & $5 \times 10^{-3}$ & $1 \times 10^{-2}$ & $1 \times 10^{-2}$ \\
$\begin{array}{l}\text { (m) } \\
\begin{array}{l}\text { Edge sizing } \\
\text { (m) }\end{array}\end{array}$ & - & $5 \times 10^{-3}$ & $5 \times 10^{-3}$ \\
$\begin{array}{l}\text { Inflation first layer } \\
\text { thickness }(\mathrm{m})\end{array}$ & $1 \times 10^{-4}$ & $1 \times 10^{-4}$ & - \\
\hline
\end{tabular}




\subsubsection{Numerical Setup}

Since Mach number is less than 0.3, the flow was considered incompressible, and pressure-based solver was utilized for simulations. Properties of the fluid are presented in Table 3. As it was investigated by Lei (2005), Spalart-Allmaras turbulence model was selected for this study since it is time efficient and valid for wing simulation at low speeds, which in this case the wings are the spoilers. In order to be able to have a valid comparison, the Reynolds number ( $\mathrm{Re}$ ) was remained at $2 \times 10^{5}$ throughout the investigations with Re corresponding to the reference length of the test model. The reference length for the spoiler was the chord. As for the ERC model, it was the length of the car. The reference area for the spoiler and the ERC model were the top area as it is the length of the car. The reference area for spoiler and the ERC model were the top area as it is presented in Table 4. The spoilers were investigated at $-6,-4,0,4$, and $8^{\circ}$ angle of attack with and without the ERC model.

Table 3. Fluid properties assumptions

\begin{tabular}{cccc}
\hline $\begin{array}{c}\text { Flu- } \\
\text { id }\end{array}$ & $\begin{array}{c}\text { Tempera- } \\
\text { ture }(\mathrm{K})\end{array}$ & $\begin{array}{c}\text { Density } \\
\left(\mathbf{k g} / \mathbf{m}^{3}\right)\end{array}$ & $\begin{array}{c}\text { Viscosity } \\
(\mathbf{k g} / \mathbf{m s})\end{array}$ \\
\hline Air & 293 & 1.225 & $1.7894 \times 10^{-5}$
\end{tabular}

Table 4. Spoiler and ERC model specifications

\begin{tabular}{lcc}
\hline & $\begin{array}{c}\text { Reference } \\
\text { length }(\mathrm{m})\end{array}$ & $\begin{array}{c}\text { Reference } \\
\text { area }\left(\mathbf{m}^{2}\right)\end{array}$ \\
\hline Spoilers & 0.04 & 0.016 \\
ERC model & 0.31 & 0.035 \\
ERC model with & 0.31 & 0.035 \\
spoilers & & \\
\hline
\end{tabular}

\subsection{Experimental Method}

To validate the result of the numerical simulation, wind tunnel experiments were conducted. The ERC model and spoilers were installed inside the Taylor's University low speed wind tunnel (TUWT) test section to conduct the experiment. TUWT is an open subsonic wind tunnel (Figure 7), which has a rectangular test section with a cross section of $0.303 \times 0.303 \mathrm{~m}$ and length of $0.885 \mathrm{~m}$. A Gamma DAQ F/T transducer was utilized measure forces and moments acting on the test models.

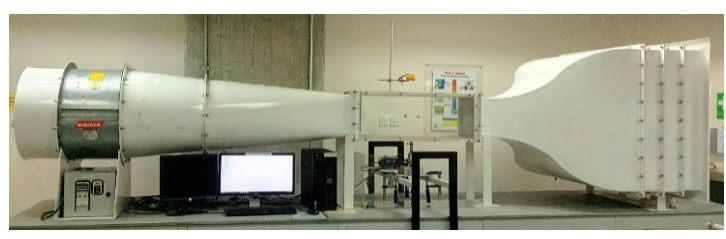

Figure 7. Taylor's University Subsonic Wind Tunnel

The same geometry with the same dimensions, which was used for the numerical simulations, were used for the experiment. The geometry of the spoilers and the ERC model was fabricated using Taylor's University 3D printing machine. The spoilers are fabricated separately and installed on the ERC model using pillars at the designated spoiler location (Figure 8).
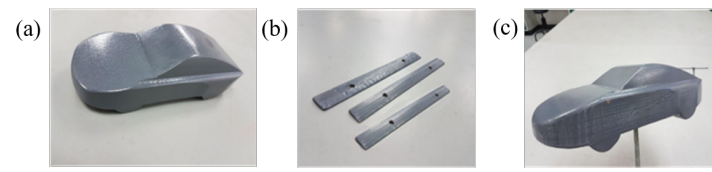

Figure 8. ERC model: (a) without spoiler, (b) 3D printed spoilers, and (c) with spoiler

\section{RESULTS AND DISCUSSION}

\subsection{AIRFOIL SELECTION}

The aerodynamic characteristics of three spoilers with NACA0012, NACA4412, and S1223 airfoil sections at eight different angles of attack (AOA) are investigated, and the simulation results are presented in Figures 9 and 10. The drag coefficient of S1223 airfoil was found to be drastically increasing as the angle of attack falls below $0^{\circ}$, while a gradual increase is observed for the NACA0012 and NACA4412. This can be explained by the higher camber of S1223 airfoil compared to other investigated airfoils. Due to such high camber, the lift coefficient of S1223 was observed to be higher than the 
other two airfoils at all investigated angles of attack. Also, NACA0012 and NACA4412 were observed to be performing similarly in terms of generated drag. However, the lift coefficient of NACA4412 was higher than NACA0012 at investigated angles by about $40 \%$. Since S1223 has negative camber, it produces higher drag. This airfoil higher pressure is produced compare to its lower surface. These characteristics are going to be reversed when these airfoils are set as a spoiler because the spoiler needs to generate downforce to makes the car more stable. Therefore, when the S1223 is set as spoiler, it generates the highest negative lift (downforce) in negative setting angles compare to NACA4412. However, the characteristics of NACA0012 were not changing since the airfoil is symmetric.

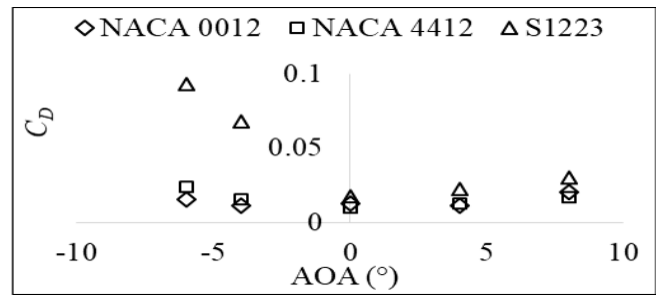

Figure 9. Drag Coefficient vs AOA in 3 different spoilers

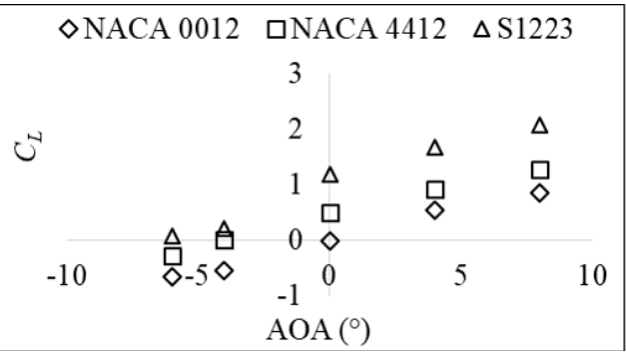

Figure 10. Lift coefficient vs AOA of 3 different spoilers

\subsection{EFFECT OF SPOILER ON THE ERC MODEL}

The velocity distribution of the flow around the ERC model with and without the spoilers is shown in Figure $\mathbf{1 1}$ for comparison. The green area behind the model indicated the low speed area, and as it observed, the rear low speed region of the models with spoiler is significantly bigger than the model without spoiler. Based on the Bernoulli's principle, reduction in speed results in pressure increment. Hence, having low speed regions behind the car can be translated into having favorable pressure, which pushes the car forward, and eventually resulting in higher speed for models with spoiler.

Figure 11 clearly shows that ERC model with S1223 has slightly higher green area at the rear of the car compare to the model with NACA4412 and NACA0012, which states that the ERC model with $\mathrm{S} 1223$ would have higher speed compare to the other 3 models. In other words, negative cambered spoilers would have a better performance compare to positive camber and symmetric airfoils.

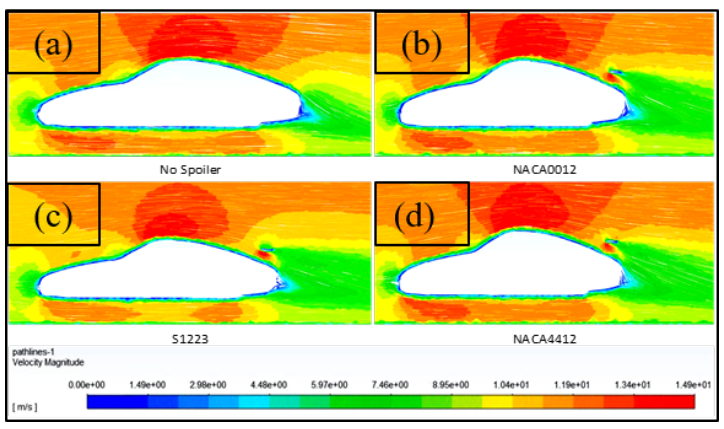

Figure 11. Velocity distribution around the ERC model without and with different spoiler configurations

The aerodynamic characteristics of the investigated test models are predicted using numerical simulations, shown in Figures $\mathbf{1 2}$ and 13. It is shown that the drag coefficient for ERC model with all of these spoilers are nearly the same. The model with $\mathrm{S} 1223$ airfoil is observed to have lowest drag compared to the test models with NACA0012 and NACA4412 spoilers. Hence, using the S1223 airfoil for spoilers helps in reducing the car overall drag which eventually results in higher speed and efficiency of the car as less energy is 
required to overcome the drag. However, observation of Figure $\mathbf{1 3}$ shows that the ERC model with S1223 airfoil at $-8^{\circ}$ angle of attack has a lift coefficient of 0.011 which is the highest among the investigated models and use of high lift configurations is strongly not advised as the amount of generated lift may result in instability and overturn of the car as it was experienced during the Le Mans race in 1999. Hence, it is suggested to use the spoiler configuration with the $\mathrm{S} 1223$ airfoil at $6^{\circ}$ angle of attack, which promises more stability, maneuverability, and safety with a small tradeoff between the stability and slight increase in generated drag.

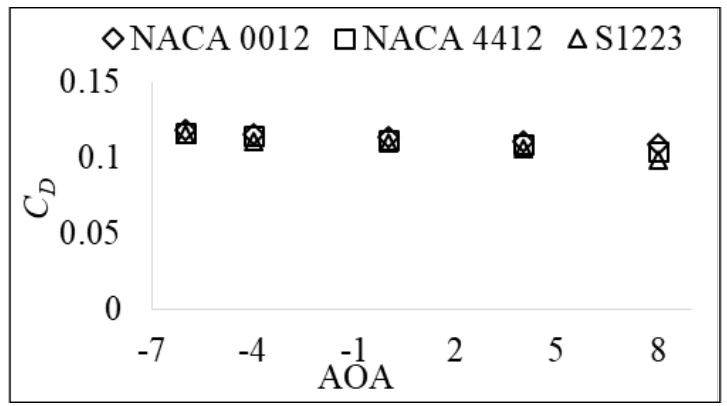

Figure 12. Drag coefficient of ERC model with spoilers in different angles of attack

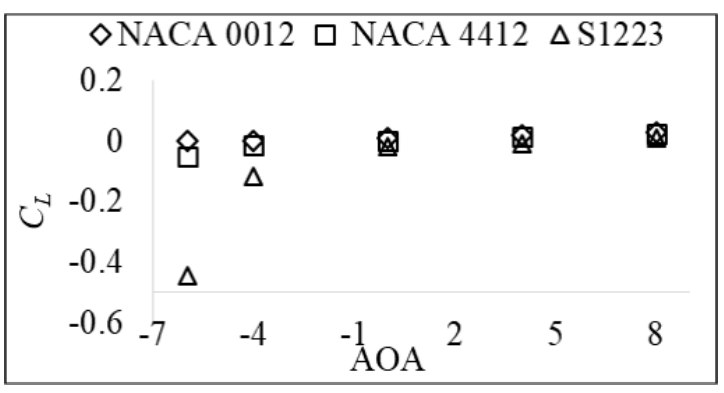

Figure 13. Lift coefficient of ERC model with spoilers in different angles of attack

\subsection{RESULTS VALIDATION}

The numerical meshing method boundary conditions were verified, and the simulation results obtained for NACA0012 at Re $2 \times 10^{5}$ and angles of attack ranging from -12 to 12 were compared to published results (see Figure 14) (Fackrell \& Harvey, 1972; Eftekhari \& Al-Obaidi, 2019). Evaluation of the drag polar shows that the results from the numerical simulation are complementing the published results at all angles of attack except 12 and $-12^{\circ}$ with a maximum percentage error of 4 and $6 \%$, respectively. Such low percentage error shows that boundary conditions are selected in the appropriate manner. Enhancing the mesh configuration can help in further reduction of the errors. Mesh configuration enhancements were achieved by refining the mesh near the model's curvature and reducing the first layer thickness of the cells to capture the boundary layer with a better resolution.

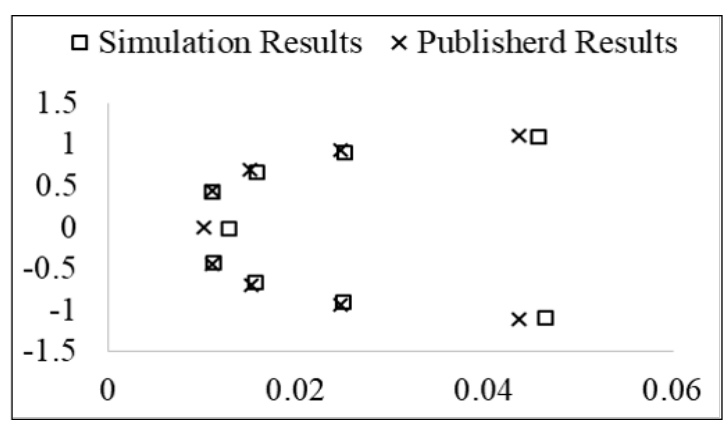

Figure 14. NACA0012 Drag Polar at Re $2 \times 10^{5}$

\subsection{VALIDATING NUMERICAL RESULT OF ERC MODEL WITH AND WITHOUT SPOILERS}

Wind-tunnel experiments were conducted on ERC model with and without spoilers with spoilers at $0^{\circ}$ angle of attack to assess the validity of the numerical investigation, and the results are shown in Figure 15. The experimental results were complementing the numerical ones. Since this is just a simulation to find the value of the lift and drag coefficient on different spoiler setting angles and shapes, the value of lift coefficient and drag coefficient are variable. But, this application in race is different since during a race the shape of the car and spoiler are fixed as well as the setting angle for the spoilers. So, during a race the velocity of the car will be variable and that will have the effect on the lift and drag force of the car. It is in the duty of an engineer to 
design the geometry of the car, and its spoiler in a way that the race car generates enough down force and reduces the drag force in a way that the race car firstly have a good stability and handling. From other side, it would be fast enough to be able to overtake other race cars during a race. Since S1223 is generating negative lift on the vehicle, it can be said that the negative cambered wings would be more suitable to use for race cars because they generate negative lift that provides the stability and handling for the race car which helps the driver to take a corner at a higher speed, which overall it will reduce the lap time. So, by applying a S1223 spoiler on ERC model on $-6^{\circ}$ setting angle, the car would have a better aerodynamic performance compared to NACA 4412 and NACA 0012 in the setting angles in the range of -6 to $8^{\circ}$. Since this ERC model was inspired by Porsche 911 and the shape of the vehicle is similar to it, S1223 at $6^{\circ}$ setting angle would also have a better aerodynamic efficiency during a race compare to a Porsche 911 without spoiler, with NACA 4412, and NACA 0012 spoiler.

\section{CONCLUSION}

In this research paper, numerical, and experimental studies are conducted on different spoiler shapes at different setting angles. The cases of ERC model with and without spoiler at different setting angles with same Reynold's number of $2 \times 10^{5}$ are investigated and analysed. Based on the achieved results, it is concluded that, utilizing spoilers has significant effect on lift and drag coefficient of the racing cars; hence the aerodynamic performance of racing cars in terms of stability and efficiency is affected due to the change in pressure distribution of the flow towards the rear end of the car. Also, it is found that S1223 as spoiler on a race car generates higher downforce compare to NACA 0012 and NACA 4412, which creates more stability for the race car. On top of that, the drag coefficient of the ERC model with S1223 spoiler is lower than the other investigated models, which leads to higher speed of the racing car, so this would state that it is better to use negative cambered airfoils as spoiler compare to positive cambered or symmetric airfoils.

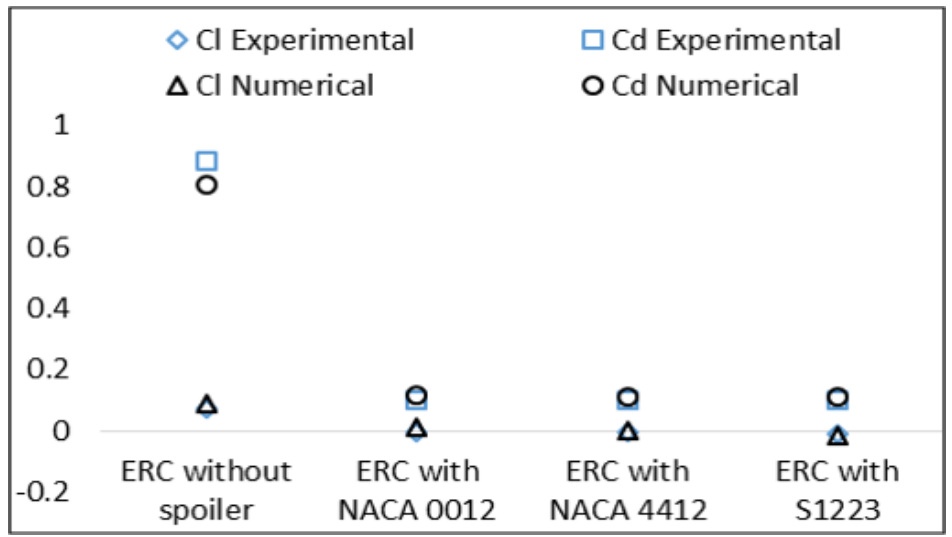

Figure 15. Lift and drag coefficient of ERC model without spoiler and with spoilers at 0-degree 
It is also observed that single-airfoiled spoilers have to be set in negative angle on the race cars in order to have a better aerodynamic stability and increasing the setting angle from negative to positive results in significant increment of the lift force, which reduces the stability and handling of the race car. Lastly, it is found that to obtain the overall drag or lift coefficient for a car with spoiler, it is not recommended to add the drag or lift coefficient of the spoiler with the drag or lift coefficient of the car model since the air flow received by spoiler is considered as disturbed flow and not fresh air.

\section{ACKNOWLEDGEMENTS}

This research was financially supported by Taylor's Research Grant Scheme (TRGS), School of Engineering, Taylor's University through Research Grant No: TRGS_MFS_2_2016_SOE_010.

\section{AUTHORS' NOTE}

The authors declare that there is no conflict of interest regarding the publication of this article. Authors confirmed that the data and the paper are free of plagiarism.

\section{REFERENCES}

Buljac, A., Džijan, I., Korade, I., Krizmanić, S., \& Kozmar, H. (2016). Automobile aerodynamics influenced by airfoil-shaped rear wing. In International journal of automotive technology, 17(3), 377-385.

Das, R. C., \& Riyad, M. (2017). CFD analysis of passenger vehicleat various angle of rear end spoiler. Procedia engineering, 194, 160-165.

Eftekhari, S., \& Al-Obaidi, A. S. M. (2019). Investigation of a NACA0012 Finite Wing Aerodynamics at Low Reynold's Numbers and $0^{\circ}$ to $90^{\circ}$ Angle of Attack. Journal of Journal of Aerospace Technology and Management, 11.

Fackrell, J. E., \& Harvey, J. K. (1972). The Flow Field and Pressure Distribution of an Isolated Road Wheel. in Advances in Road Vehicle Aerodynamics.

Heisler, H. (2007). Vehicle body aerodynamics. Advanced Vehicle Technology.

Lei, Z. (2005). Effect of rans turbulence models on computation of vortical flow over wingbody configuration. Transactions of the Japan Society for Aeronautical and Space Sciences, 48(161).

Reddy, P. R., \& Lokanadham, R. (2017). Cfd Analysis on Aerodynamic Effects on a Passenger Car. International Research Journal of Engineering and Technology, 4(9).

Su, Y., \& Basavaraj, A. (2017, July). Computational Analysis of Benzing Airfoils for Optimization in a Wing Configuration for a Formula SAE Car. In ASME 2017 Fluids Engineering Division Summer Meeting. American Society of Mechanical Engineers Digital Collection.

Vadgama, T. N., Patel, A., Thakkar, D., \& Vala, J. (2015). Structural Analysis of Formula One Racing Car. International Journal of Advance Research in Engineering, Science \& Technology, 2(5). 
Hesam Eftekhari, et al. The Effect of Spoiler Shape and Setting Angle on Racing Cars...| 20

Walter, D. J. (2007). Study of Aerofoils at High Angle of Attack in Ground Effect. Manufacturing Engineering.

Yew, L. W., Al-Obaidi, A. S. M., \& Namasivayam, S. (2017). Design and Development of a Multi-Element Active Aerodynamic Package to Enhance the Performance of Taylor's Formula SAE Car. in International Engineering Research Conference (8th Eureca). 